\title{
Non-Contact Human Body Parameter Measurement Based on Kinect Sensor
}

\author{
A. M. S. B. Adikari ${ }^{1}$, N. G. C. Ganegoda ${ }^{2}$, W. K. I. L. Wanniarachchi ${ }^{1 *}$ \\ ${ }^{1}$ (Department of Physics, Faculty of Applied Sciences, University of Sri Jayewardenepura, Sri Lanka) \\ ${ }_{2}^{2}$ (Department of Mathematics, Faculty of Applied Sciences, University of Sri Jayewardenepura, Sri Lanka) \\ *Corresponding Email: iwanni@sjp.ac.lk
}

\begin{abstract}
In this work we implemented a system to obtain human body parameter measurement without physically contacting the user. This implementation is contained the methods of obtaining $3 D$ measurements using Kinect $v 2$ depth sensor. The developed system at the initial stage is capable of detecting and obtaining personalized body parameters such as height, shoulder length, neck to hip length, hip to leg length and arm length by incorporating the necessary skeleton joints and front perimeter at chest, stomach and waist by incorporating the necessary $3 D$ pixels. According to the results, the measurement on height and arm length of the person are relatively in good agreement with the actual values since the error is less than $5 \%$ and measurement has been taken in centimeters. Maximum $12 \%$ of an error incorporated of calculating front perimeter at chest. Experimental results obtained from the developed system are in acceptable range for dressing purpose and ultimately helpful for designing a real time $3 D$ virtual dressing room.
\end{abstract}

Keywords: Human Body Measurement, Kinect Sensor, Virtual Dressing Room (VDR), Augmented Reality

\section{Introduction}

Real-time augmented reality is now one of the most trending topics [1] in virtual simulation field. The virtual dressing room (VDR) is designed using the techniques of augmented reality which can be used for retailer shops to give a better customer experience. The best judgment on a dress can be done by wearing it physically, however, this need some time and space for individual customer. Therefore, it is not easy and feasible from customer and retailer sides especially during festive seasons. The concept of virtual dressing room is introduced in order to overcome this problem [2].

Several methods are already implemented in concepts level but it has been more difficult to make it production level since the outputs are not realistic as expected [3]. To obtain correct body measurements of a person is one of the main issues faced by researchers [4]. Also, the developed system outputs were not in three dimensional (3D) and lack of dynamic processing where it hard to compute and costly [5]. Earlier it has been used a single camera [6], camera array [7] and also with Kinect depth sensor version one [8]. All these methods have been implemented using image processing based and using static images with 2D outputs.

Using a single web camera and camera array based virtual dressing the image processing should be done by the application host computer. It consumed lot of computer resources while doing the image processing and virtual dressing part of the application. This might be caused to the considerable lag creation for the final output. By using a single camera only it is difficult to compute the depth information according to the paper "Virtual TryOn through Image-Based Rendering by S. Hauswiesner, Matthias Straka, and Gerhard Reitmayr" [2]. The selfimage processing units such as Kinect depth sensor can be used to reduce above mentioned lag at the output [9] .

In this research it has mainly focused on to the detecting of the 3D measurements and gesture movement identification of the human body using the Kinect $\mathrm{v} 2$ sensor for real-time 3D virtual dressing room. This Kinect device is contained few sensors to identify the color and depth information which are necessary for the 3D calculations and it is faster computing images over general camera module. The Kinect is equipped with RGB sensor, Depth, Infrared sensor and microphone array. Also it has an inbuilt processor and algorithm to manipulate the human body data such as skeleton. Comparing Kinect v1, the Kinect v2 can be identify up-to 6 people with 25 joins for each. And the accuracy of the image processing of the Kinect v2 is higher than the Kinect v1 [10]. The system developed at the initial stage is capable of detecting and obtaining personalized body parameters such as height, shoulder length, neck to hip length, hip to leg length and arm length by incorporating the necessary skeleton joints and front perimeter at chest, stomach and waist by incorporating the necessary 3D pixels.

\section{Methodology}

The body (skeleton and body index image with colors) was identified by the Kinect v2 device. When a person stands in front of the sensor, it captures color image and the depth image of the person using the RGB, IR and depth sensors. Then the background noise and moving objects are identified using image processing 
techniques in the sensor middleware [11]. The depth information of the user's body was identified according to the user position. Using the sensor middleware the skeleton positions and orientations were identified. Basic noise reduction was done by the Kinect sensor. The following figure 1 indicates the flow of events when obtaining the user body measurements.

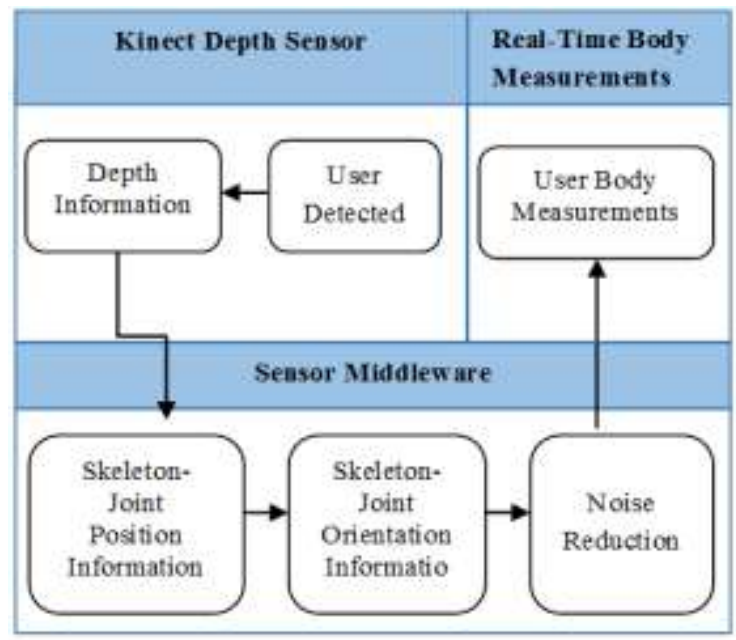

Fig. 1 Kinect sensor operation to obtain user body measurements

In the VDR concept a premade dress is fitted on to the 3D model of the customer [12]. The Kinect sensor unit used in this project has the capacity to store 25 skeleton points of a person as illustrated in the figure 2 [13].

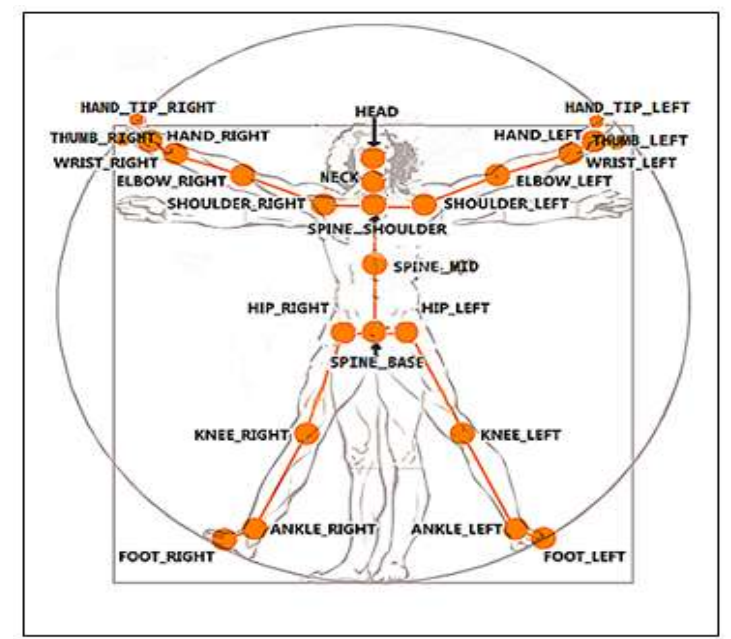

Fig. 2 Davin chi pose of the Kinect joint mapping- 25 skeleton points

Also the same Kinect sensor can extend this database to store skeleton points of six people simultaneously. Therefore, following parameters are identified as vital to user body measurements of the customer. For these measurements it has only used single person with "T" pose as shown in figure 3.

1. Height of the user - Head to ankle (mean value of spine-base to ankle left and right).

2. Shoulder length - Shoulder right to shoulder left.

3. Neck to hip length - Neck to spine-base.

4. Hip to leg length - Spine-base to ankle (mean value of spine-base to ankle left and right).

5. Arm length - Shoulder left to wrist left (mean value of both right and left).

6. Chest circumference (front end) - Average front perimeter of the chest.

7. Stomach circumference (front end) - Average front perimeter around stomach area.

8. Waist circumference (front end) - Average front perimeter of the waist. 


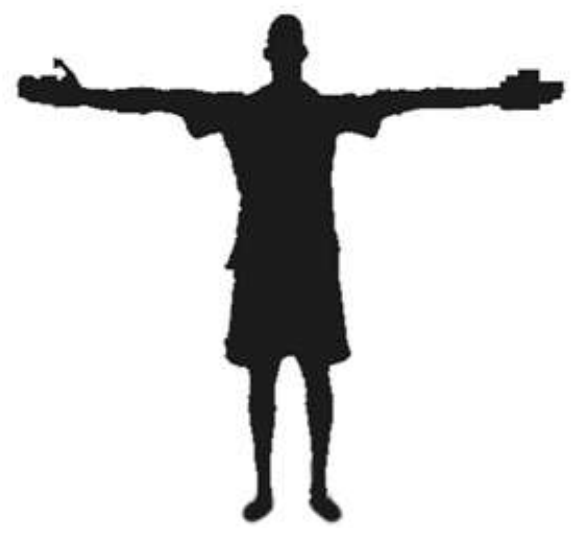

Fig. 3 3D model with "T" pose captured with Kinect

\subsection{Measurements incorporate with skeleton points}

For the measurements such as height, shoulder length, neck to hip length, hip to leg length and arm length, the appropriate skeleton point's distances were taken in to account using direct Kinect application programming interfaces (APIs). The calculations for above mentioned parameters were obtained using the Pythagoras theorem for 3D space. Using the Kinect it can be obtained $\mathrm{x}, \mathrm{y}$, and $\mathrm{z}$ coordinates of each joint point (skeleton point) as in figure 2. C\# code was developed for all calculation in this work. The following example illustrates the calculation of the distance of two points.

Shoulder left point $\left(\mathrm{x}_{1}, \mathrm{y}_{1}, \mathrm{z}_{1}\right)$ to spine shoulder point $\left(\mathrm{x}_{2}, \mathrm{y}_{2}, \mathrm{z}_{2}\right) .(0,0,0)$ coordinates are defined at the center of the camera space. Here $\mathrm{z}=0$ position is the focal point of the camera. Distance between two points;

$$
\left[\left(\mathrm{x}_{1}-\mathrm{x}_{2}\right)^{2}+\left(\mathrm{y}_{1}-\mathrm{y}_{2}\right)^{2}+\left(\mathrm{z}_{1}-\mathrm{z}_{2}\right)^{2}\right]^{1 / 2}
$$

Using equation (1) the distance between point one and spine shoulder can be obtained $\left(\mathrm{d}_{1}\right)$. Using above way shoulder right and spine shoulder can be obtained $\left(d_{2}\right)$. Using $d_{1}$ and $d_{2}$ values shoulder length parameter $(d=$ $\mathrm{d} 1+\mathrm{d} 2)$ can be determined. For the height of the user head, neck, spine shoulder, spine mid, spine base, knee right/left and ankle right/left skeleton points were taken in to account. For hip to leg length of the user spine base, knee right/left ankle right/left skeleton points were considered. In necessary cases average values were obtained for more accurate results.

Similarly, all other calculated parameters were added to an array and a comparison was made relative to manual measurements taken on the same person. Finally, the skeleton model was viewed using view-box in C\# and the necessary parameters were recorded for the 3D model on label objects. These measurements can be used for constructing the 3D model.

Error calibration was added for each distance using set of known measurement due to the un-calibrated results from the Kinect device. In the initial calibration step, data were collected from two users. For each of the users the necessary body parameters for dressing were manually measured. Then the sensor based calculated values for each body parameters were obtained. Accordingly, average error percentage for each measurement was obtained as in equation 2. Error;

\section{calculated value based on skeleton points - manual measurement}

manual measurement

The more accurate value for body parameter measurement was obtained as in equation 3 .

Final value;

$$
\text { Calculated value }+(\text { Calculated value } \times \text { Error percentage })
$$

\subsection{Measurements incorporate with pixels}

When measuring chest, stomach and waist perimeters of a user, the skeleton points cannot be taken into account as explained in section A. To overcome this, depth frames and pixel based measurements were used by converting depth pixel into real world camera space points. As for the figure 4 the pixel distances for chest (A), stomach (B) and waist (C) were taken into account. The y coordinate for each case A, B and C is a constant. For $\mathrm{X}$ and $\mathrm{z}$ (depth) coordinates, all the color pixels from left to right and depth information incorporate with each color pixel were taken into account respectively. Using $\mathrm{x}, \mathrm{y}$ and $\mathrm{z}$ coordinate at each pixel point, it can be calculated the 


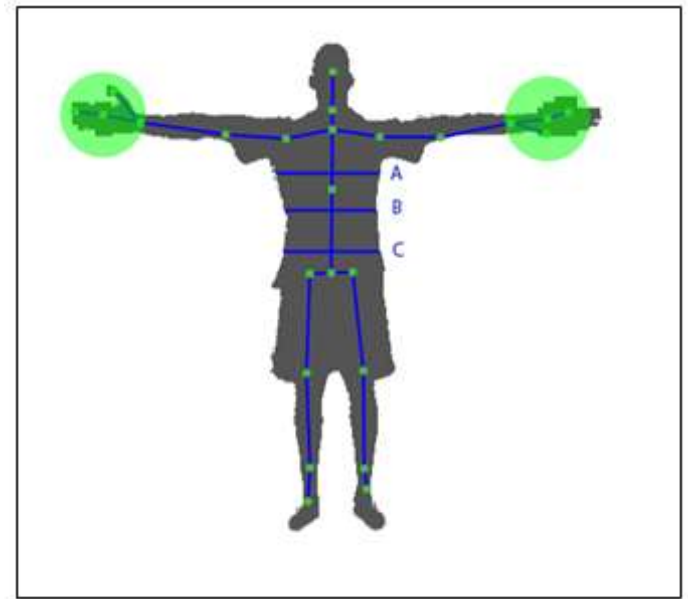

Fig. 4 Measurement taking from "T" pose incorporate with pixels

Front end (facing to the Kinect sensor) perimeter for each case A, B and C of a person who in-front of the Kinect device. The y position of A was taken one forth distance of the distance between spine-shoulder and spine-mid skeleton points, below the spine-shoulder point. The y position of B was taken one half distance of the distance between spine-mid and spine-base points, below the spine-mid skeleton point. Three quarter distance of (|spine mid - spine base|), below the spine mid skeleton point was taken as y position of measurement of waist (C). Pixel to pixel distance calculated according to the Pythagoras theorem in 3D space and summation of all individual distance of the front end pixels relevant to $\mathrm{A}, \mathrm{B}$ and $\mathrm{C}$ were assigned as front side perimeter at each level. The final conversion would be color frame $\rightarrow$ depth frame $\rightarrow$ camera space frame. For the conversion color frame has 1920 x 1080p resolution and depth frame has 512 x 424 resolutions [14]. Camera space conversion can be done by the internal API includes with Kinect software development kit (SDK).

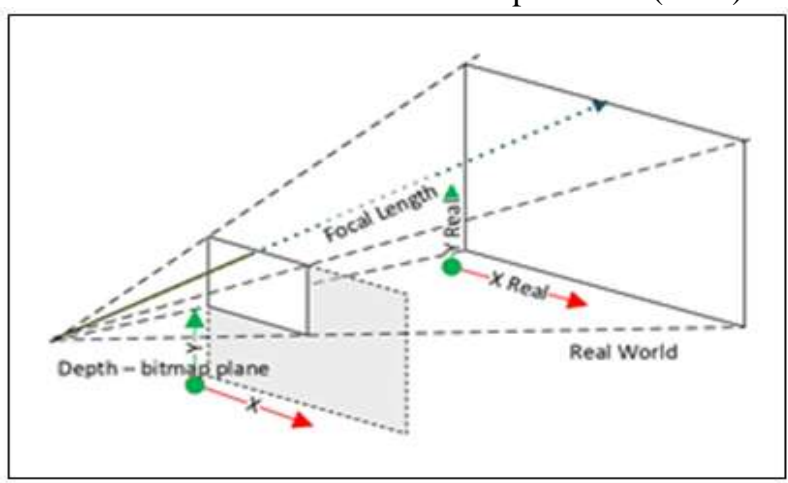

Fig. 5 Color and depth image to real world distance conversion

Figure 5 shows the conversion of color frame to real world (camera space frame) using depth frame and color frame information. This is help to convert the pixel value data to real world measurement meters.

\section{Results And Discussion}

The Table 1 compares the measurements on the person obtained using the Kinect sensor and manual measurements for each length such as height, shoulder length, neck to hip length, hip to leg length and arm length. The front end perimeter of chest, stomach and waist are also shown in Table 1. The second column of the table 01 listed the calculated values as of the method explained under section A. As the user is not a perfectly stable, when calculating the shoulder length the maximum distance for the shoulder length was considered. According to the results, the sensor measured parameters as per the equation 2 are relatively in good agreement with the manually measured values for length measurements in $\mathrm{cm}$ units. The results for font end chest, stomach and waist perimeters incorporate maximum of $12 \%$ error where these calculations need of improve methods to reduce the error. When calculating these front and perimeter at each level, the equation 2 was not considered. These values are calculated as mentioned in section B in the methodology. The average weighted sampling technique has been accounted to the each calculation to minimize the error incorporated in all the parameters discussed in this work. The developed system updates current values taken from the sensor at each $50 \mathrm{~ms}$. 
Correction for the each sample;

$\left[\left(\right.\right.$ Final Value $\left._{\mathrm{n}} \times 0.1\right)+\left(\right.$ Final Value $\left.\left._{\mathrm{n}-1} \times 0.9\right)\right]$

Using above equation (4) it has minimized the variation that can be occurred to the final value due to outliers.

Table 1 Body parameter measurements

\begin{tabular}{|c|c|c|c|}
\hline Measurement & $\begin{array}{c}\text { Manually } \\
\text { Measured } \\
\text { Value }(\mathrm{cm})\end{array}$ & $\begin{array}{c}\text { Sensor Measured } \\
\text { Value }(\mathrm{cm}) \\
\text { Eq. }(2)\end{array}$ & $\begin{array}{c}\text { Error } \\
{\left[\frac{|\Delta x|}{x} \times 100 \% \delta\right]}\end{array}$ \\
\hline Height of the user & 185.9 & 186.0 & 0.05 \\
\hline Shoulder length & 45.6 & 47.2 & 3.60 \\
\hline Neck to hip length & 78.0 & 79.4 & 4.82 \\
\hline Hip to leg length & 79.9 & 77.5 & 3.00 \\
\hline Arm length & 55.9 & 53.2 & 4.80 \\
\hline Chest front end perimeter & 51.0 & 56.9 & 11.60 \\
\hline $\begin{array}{l}\text { Stomach front end } \\
\text { perimeter }\end{array}$ & 50.5 & 55.6 & 10.20 \\
\hline Waist front end perimeter & 43.2 & 47.9 & 10.97 \\
\hline
\end{tabular}

$|\Delta \mathrm{x}|=$ Absolute value of [Sensor Measured - Manually Measured]

Further, gesture controlling system was introduced in this work to control the system without using any other input devices such and keyboards, mouse etc... The implementation of the gesture controlling system mainly focused on up, down, left and right directions and record, save, and reset features were also embedded to the system. For the gesture identification using Kinect and SDK there are several APIs. Microsoft Visual gesture builder was used for this purpose. Initially it was needed to recorded few gesture pattern and recorded in to a (.gbd) database. Then using the main-code navigation and selections gesture controlling system was implemented without any input device.

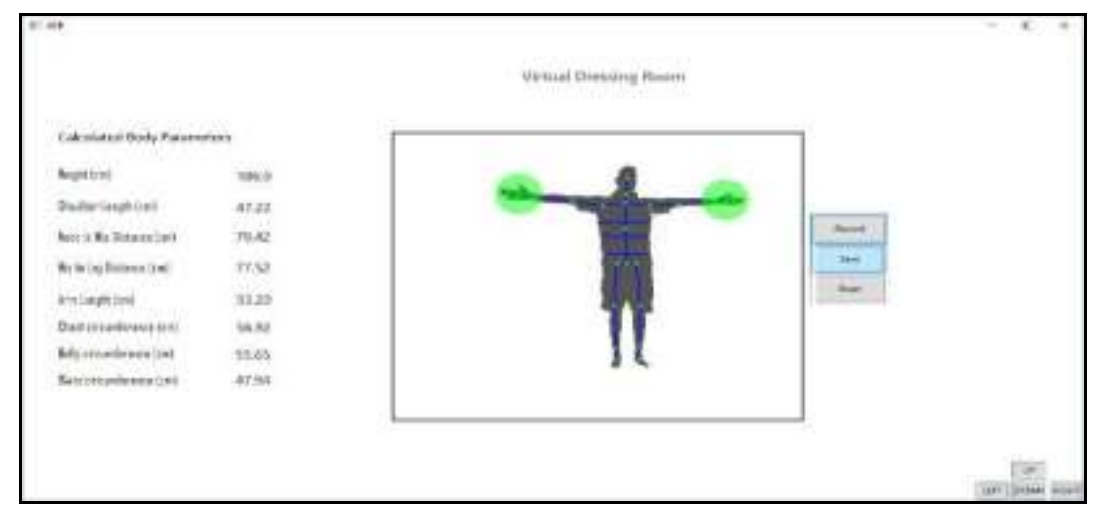

Fig. 6 Graphical user interface for VDR

The figure 6 shows the user interface of the VDR developed in this work. The interface has three control buttons for recording, saving, and resetting where enable user to record the images while rotating in front of the Kinect sensor. Once recording is saved, the user skeleton image is displayed with above mentioned body parameter measurements of the person. Figure 7 shows the top view of perimeter measurements of chest, stomach and waist of a user. Vertical axis represents the depth information in $\mathrm{cm}$ and $\mathrm{x}$ axis represents the distance in $\mathrm{cm}$ from left to right at each level A, B and C.

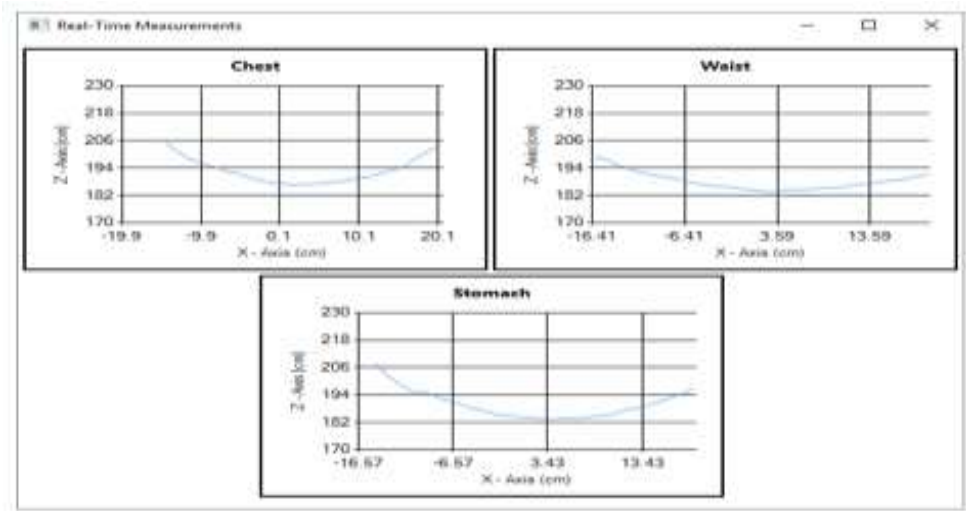

Fig. 7 Top view of depth (z) vs horizontal (x) distance incorporated with equation (4), (a) Chest front end (b) Stomach front end and (c) Waist front end 


\section{Conclusion}

The system developed at the initial stage is capable of detecting and obtaining personalized body parameters such as height, shoulder, neck to hip, hip to leg, and arm length and the front end perimeter of chest, stomach and waist. The errors reported for the calculated and actual values are minimum suggesting that the Kinect sensor can be used to develop a virtual dressing room (VDR).

According to the error percentages all the values were around $5 \%$ for the lengths. But it shows around $12 \%$ errors for perimeter measurements for chest, stomach and waist where these measurements need of an improve method of calculation.

Using two Kinect depth sensors at front and back side of the user can minimize the error incorporated with chest, stomach and waist measurements.

\section{Acknowledgements}

Authors wish to acknowledge university research grant ASP/01/RE/SCI/2015/27 supported for this project.

\section{References}

[1] Feng Zhou, H. B. L. Duh and M. Billinghurst, "Trends in augmented reality tracking, interaction and display: A review of ten years of ISMAR," 2008 7th IEEE/ACM International Symposium on Mixed and Augmented Reality, Cambridge, 2008, pp. 193-202.

[2] S. Hauswiesner, Matthias Straka, and Gerhard Reitmayr, Virtual Try-On through Image-Based Rendering, IEEE Transactions On Visualization And Computer Graphics, Vol. 19, No. 9, September 2013

[3] Boonbrahm, Poonpong, Charlee Kaewrat, and Salin Boonbrahm. "Realistic Simulation in Virtual Fitting Room Using Physical Properties of Fabrics." Procedia Computer Science 75 (2015): pp. 12-16.

[4] M. Kasap and N. Magnenat-Thalmann, Parameterized human body model for real-time applications, CW '07. International Conference on Cyberworlds, IEEE Computer Society, 2007, pp. 160-167.

[5] Walter, Bruce, et al. "Fitting virtual lights for non-diffuse walkthroughs." Proceedings of the 24th annual conference on Computer graphics and interactive techniques. ACM Press/Addison-Wesley Publishing Co., 1997.

[6] Chinmay Barde, Soham Nadkarni, Nikhil Joshi and Saurabh Joshi, Virtual Fitting Room using Webcam, International Journal of Engineering and Technical Research (IJETR) ISSN: 2321-0869, Volume-3, Issue-3, March 2015

[7] S. Hauswiesner, Matthias Straka, and Gerhard Reitmayr, Virtual Try-On through Image-Based Rendering, IEEE Transactions On Visualization And Computer Graphics, Vol. 19, No. 9, September 2013

[8] F. Is,1kdo gan and G. Kara, A real time virtual dressing room application using Kinect, CMPE537 Computer Vision Course Project, January 2012

[9] I. Pachoulakis and K. Kapetanakis, "Augmented reality platforms for virtual Fitting rooms", The International Journal of Multimedia \& Its Applications (IJMA) Vol.4, No.4, August 2012

[10] S. Zennaro et al., "Performance evaluation of the 1st and 2nd generation Kinect for multimedia applications," 2015 IEEE International Conference on Multimedia and Expo (ICME), Turin, 2015, pp. 1-6.

[11] L. Yang, L. Zhang, H. Dong, A. Alelaiwi and A. E. Saddik, "Evaluating and Improving the Depth Accuracy of Kinect for Windows v2," in IEEE Sensors Journal, vol. 15, no. 8, pp. 4275-4285, Aug. 2015.

[12] S. Hauswiesner, M. Straka and G. Reitmayr, "Image-based clothes transfer," 2011 10th IEEE International Symposium on Mixed and Augmented Reality, Basel, 2011, pp. 169-172.

[13] JointType Enumeration Available at (https://msdn.microsoft.com/en-us/library/microsoft.kinect.jointtype.aspx) [accessed on January 2017].

[14] Kinect Hardware at (https://developer.microsoft.com/en-us/windows/kinect/hardware) [accessed on January 2017].

[15] I. Samejima, K. Maki, S. Kagami, M. Kouchi, and H. Mizoguchi “A Body Dimensions Estimation Method of Subject from a Few Measurement Items Using KINECT," in Proceedings of IEEE International Conference on Systems, Man, and Cybernetics, SMC '2012, pp. 3384\{3389, IEEE, 2012.

[16] A. L. Brooks and E. Brooks, "Towards an inclusive virtual dressing room for wheelchair-bound customers," 2014 International Conference on Collaboration Technologies and Systems (CTS), Minneapolis, MN, 2014, pp. 582-589.

[17] R. Nakamura, M. Izutsu and S. Hatakeyama, "Estimation Method of Clothes Size for Virtual Fitting Room with Kinect Sensor," 2013 IEEE International Conference on Systems, Man, and Cybernetics, Manchester, 2013, pp. 3733-3738. 